The term proteomics was used for the first time in 1995 to describe large-scale protein analyses. At the same time proteomics was distinguished as a new domain of the life sciences. The major object of proteomic studies is the proteome, i.e. the set of all proteins accumulating in a given cell, tissue or organ. During the last years several new methods and techniques have been developed to increase the fidelity and efficacy of proteomic analyses. The most widely used are two-dimensional electrophoresis (2DE) and mass spectrometry (MS).

In the past decade proteomic analyses have also been successfully applied in biomedical research. They allow one to determine how various diseases affect the pattern of protein accumulation. In this paper, we attempt to summarize the results of the proteomic analyses of acute myeloid leukemia (AML) cells. They have increased our knowledge on the mechanisms underlying AML development and contributed to progress in $\mathrm{AML}$ diagnostics and treatment.

Key words: acute myeloid leukemia, pro teomics, 2DE, mass spectrometry.

\section{Comparative proteomics in acute myeloid leukemia}

\author{
Magdalena Luczak ${ }^{1}$, Maciej Kaźmierczak², Luiza Hadschuh'12, \\ Krzysztof Lewandowski', Mieczysław Komarnicki ${ }^{2}$, Marek Figlerowicz ${ }^{1,3}$
}

1Institute of Bioorganic Chemistry, Polish Academy of Sciences, Poznan, Poland 2Department of Hematology, Poznan University of Medical Sciences, Poznan, Poland 3 Institute of Computing Science, Poznan University of Technology, Poznan, Poland

\section{Introduction}

Acute myeloid leukemia (AML) is a malignant, clonal, highly genetically heterogeneous disease of blood-forming tissue. AML is characterized by impaired production of normal morphotic blood elements, accumulation of abnormal cells called blasts and invasion of various organs and tissues. For many years, AML diagnostics was mainly based on classic laboratory blood tests (morphological and cytochemical assessment of leukemic cells). On the basis of these tests, the French-American-British AML classification was created, which makes it possible to distinguish eight basic subtypes of the disease (AML MO-M7) (Table 1) [1]. The currently used AML classification system was proposed in 2008 by the World Health Organization (WHO). It considers the morphological features of leukemic cells, their immunophenotype and the results of cytogenetic and genetic analyses (Table 2) [2]. Its introduction resulted in increasing the number of AML subtypes to approximately 30 . The new AML classification is of significant prognostic importance; however, it practically does not affect the therapeutic patterns. Uniform treatment is recommended in the majority of cases, which is not appropriate with the multitude of AML subtypes. In addition, the clinical and laboratory test-based AML prognostic models are characterized by a low predictive value. Therefore, it is absolutely necessary to take further action to explain the molecular mechanisms that underlie leukemic transformations. Their identification would certainly contribute to the development of new strategies for AML treatment and new forms of the so-called targeted therapy. Currently, the group of basic factors inducing cancer transformation include abnormal activation of signal transduction pathways (STP), defective regulation of the cell cycle and apoptosis disorders [3, 4].

Large-scale research techniques developed in recent years (genomic, proteomic, metabolic) have created completely new possibilities of simultaneous analysis of an enormous number of factors affecting all physiological and pathological processes that occur in living systems. Solely, a comparative analysis of gene expression profiles in various AML subtypes provided a sufficient amount of information to propose a new classification system and define new subgroups of potential prognostic importance $[5,6]$. The microarray techniques used in this research made it possible to assess the level of individual mRNA accumulation. Unfortunately, DNA microarrays based methods are burdened with numerous flaws. Firstly, they are extremely complicated and costly. Additionally, the quantity of the generated transcript does not always correspond to the quantity of protein produced. Hence, it is still necessary to identify new and more reliable biomarkers. Comparative proteomic analysis is one of the most interesting and promising approaches. It facilitates the examination of very complex protein mixtures by quantitative and qualitative assessments of their individual components.

The term "proteomics" was first used in 1995 to define large-scale analyses of proteins. At the same time, proteomics was defined as a new field of 
Table 1. French-American-British AML classification

\begin{tabular}{|ll}
\hline AML subtype & Description \\
\hline M0 & AML with minimal differentiation, 3-5\% of cases \\
\hline M1 & AML without maturation, $15-20 \%$ of cases \\
\hline M2 & AML with maturation, $25-30 \%$ of cases \\
\hline M3 & acute promyelocytic leukemia (APL), $10-15 \%$ of cases \\
\hline M4 & acute myelomonocytic leukemia, $20-30 \%$ of cases \\
\hline M5a & acute monoblastic leukemia; $2-7 \%$ of cases \\
\hline M5b & acute monocytic leukemia; $2-5 \%$ of cases \\
\hline M6 & acute erythroid leukemia; 3-5\% of cases \\
\hline M7 & acute megakaryoblastic leukemia; 3-5\% of cases \\
\hline
\end{tabular}

Table 2. WHO AML classification

\begin{tabular}{|c|c|}
\hline AML subtype & Description \\
\hline $\begin{array}{l}\text { AML with recurrent } \\
\text { genetic } \\
\text { abnormalities }\end{array}$ & $\begin{array}{l}\text { AML with translocation } t(8 ; 21)(q 22 ; q 22) \\
\text { AML with inv }(16) \\
\text { AML with } 11 q 23 \text { translocations } \\
\text { AML with RAR fusions } t(15 ; 17)\end{array}$ \\
\hline $\begin{array}{l}\text { AML with } \\
\text { multilineage } \\
\text { dysplasia }\end{array}$ & $\begin{array}{l}\text { after a preceding myelodysplastic } \\
\text { syndrome (MDS) } \\
\text { without previous MDS }\end{array}$ \\
\hline AML therapy-related & $\begin{array}{l}\text { alkylating agent and radiation Therapy } \\
\text { Related AML } \\
\text { topoisomerase II Inhibitor Related AML } \\
\text { (MDS) }\end{array}$ \\
\hline $\begin{array}{l}\text { AML not otherwise } \\
\text { categorized }\end{array}$ & $\begin{array}{l}\text { AML minimally differentiated (FAB - M0) } \\
\text { AML without maturation ( } F A B-M 1) \\
\text { AML with maturation ( } F A B-M 2 \text { and } M 3 \text { ) } \\
\text { acute myelomonocytic leukemia ( } F A B-M 4) \\
\text { acute monoblastic leukemia and monocytic } \\
\text { leukemia (FAB - M5a i M5b) } \\
\text { acute erythroleukemia ( } F A B-M 6) \\
\text { acute megakarioblastic leukemia (FAB - M7) } \\
\text { acute basophilic leukemia } \\
\text { acute panmyelosis with myelofibrosis } \\
\text { myeloid sarcoma }\end{array}$ \\
\hline
\end{tabular}

science focusing on the proteome, i.e. a set of all proteins occurring at a given time and at a given place in an organism. Depending on the character and scope of research conducted, we can refer to a cellular proteome, tissue proteome or the proteome of the whole body. In the last years, a range of techniques and methods have been developed which increase the efficiency and effectiveness of proteomic analyses. These procedures include two-dimensional electrophoresis (2DE), mass spectrometry (MS), and complex chromatographic, biochemical and immunolocalization approaches. Recently, the techniques involving protein microarrays have been developed. Although, useful in both qualitative and quantitative proteome analyses they are still not very popular. By using them one can identify post-translational modifications or assess the activity of a selected set of proteins. Protein microarrays are also free of limitations of conventional techniques, such as Western blot and ELISA.

\section{Proteomics in the studies of acute myeloid leukemia pathogenesis and development}

Hematopoiesis is a series of complex, dynamic and precisely arranged processes that include self-renewal and differentiation of bone marrow stem cells. The differentiation of the common myeloid precursor cells lead to the formation of cells of granulocytic and monocytic lines (Fig. 1). Even slight disturbances within just one process may result in the development of various diseases, starting from bone marrow aplasia to myelodysplastic syndromes and leukemia. Therefore, all changes occurring during hematopoiesis are strictly regulated by signal transmission pathways and transcription networks modulating gene expression. In recent years, several epigenetic regulatory networks have also been identified. They control DNA methylation and histone modification and, in this way, shape the profiles of gene expression, both protein and microRNA encoding ones [7]. By employing high-throughput proteomic methods one can simultaneously follow all of these processes at the protein level. Thus, proteomic can play a very important role in the exploration of molecular mechanisms involved in leukemogenesis.

In AML patients, C/EBP $\alpha$-p30 mutation in the gene encoding the transcription factor $\mathrm{C} / \mathrm{EBP} \alpha$ is observed particularly frequently - in approx. $9 \%$ of patients. C/EBP $\alpha$ plays an important role in granulopoiesis as its dysfunction or absence leads to the inhibition of myeloid cell differentiation and, thus, to the development of leukemia [8, 9]. Research on myeloblasts with the mutated C/EBP $\alpha$ gene permitted the identification of several events leading to leukemic transformation. 2DE and MS analyses showed that the concentration of the Ubc9 protein increases in these cells. As a result, an effector of the mutated transcription factor which participates in the modification of C/EBP $\alpha-p 42$ protein was identified. This post-translational modification (sumoylation i.e. the attachment of SUMO protein - a small ubiquitin-like modifier), inhibits C/EBP $\alpha$-p42 at the transcription level and the process of granulocyte differentiation is stopped. Proteomic methods were also used to identify Max transcription factors. They bind with C/EBP $\alpha$ and this interaction is necessary for granulocytic line differentiation. These observations were additionally confirmed by genomic analyses. It was demonstrated that the increased expression of the Max and C/EBPa genes stimulates granulocyte differentiation, while Max gene silencing effectively inhibits this process [11]. Additionally, Pulikkan et al. [12] have recently shown that peptidyl-prolyl isomerase, PIN1, is formed in AML cells. It was found that the mutated transcription factor C/EBP $\alpha$-p30 induces the expression of the PIN1 gene. The authors showed that the suppression of the PIN1 gene leads to the myeloid blast differentiation, and its increased expression, causes the maturation block similar to observed in patients with primary AML and the C/EBP $\alpha$ mutation. It was also found that PIN1 increases the stability of the c-June protein by inhibiting its ubiquitination, and this way blocks granulocyte differentiation [12]. There are, however, several other factors that influence the granulocyte differentiation. Hahn et al. [13] discovered that inhibitors of the epidermal growth factor receptor (EGFR) induce differentiation by a mechanism unrelated to EGFR. Tsao et al. [14], suggested that the changes in cellular concentration of PPARgamma re- 


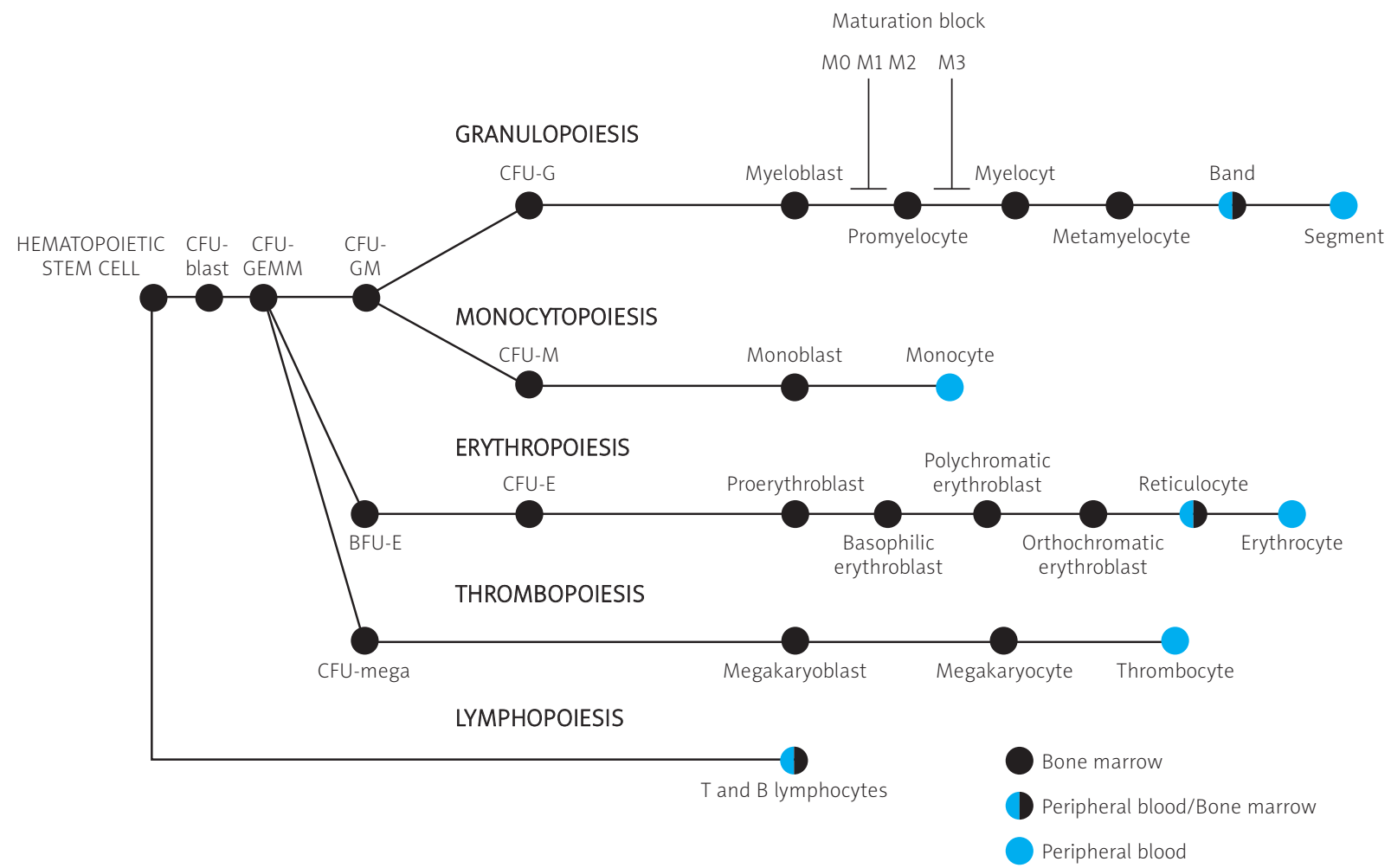

CFU-blast (colony forming unit) - a blast colony forming cell, CFU-GEMM - a cell forming a colony for erythrocytic, granulocytic, platelet and monocytic lines, CFU-GM - a cell forming a colony for erythrocytic and monocytic lines, BFU-E (base-forming unit) - another form of a cell forming a colony for an erythrocytic line, CFU-mega - a CFU cell for a platelet line, CFU-G - a CFU cell for a granulocytic line, CFU-M - a CFU cell for a monocytic line, CFU-E - a CFU cell for an erythrocytic line. The stages at which the development of cells is most likely blocked in some AML subtypes are marked. Only in the case of M1, M2 and M3 AML subtypes is there a sufficient amount of data to suggest the developmental block location.

Fig. 1. Hematopoiesis diagram

ceptor influence the induction of apoptosis by caspase-8 activation. Additionally, they postulated that the DRIP205 protein (vitamin D-interacting protein 205), which functions as a PPAR $\gamma$ co-activator, is also pivotal determinant of differentiation.

Carcinogenesis is often initiated by changes in the pattern or level of protein phosphorylation. STAT5 (STAT - signal transducers and activators of transcription) are such proteins which undergo constitutive phosphorylation in AML cells. In some cases, phosphorylation of these proteins correlates with the occurrence of mutations in FLT3 and KIT genes. This phenomenon has recently been quite well explored $[15,16]$. However, the mechanism of STAT5 protein phosphorylation is still unclear in the case of lack of mutation in FLT3 and KIT genes, which is observed in $35 \%$ of patients. This phenomenon was investigated in AML cell lines using the immunoprecipitation, technique involving monoclonal antibodies specific for phosphotyrosine, and MS for protein identification [17]. As a result, janus kinase (JAK2) was identified as the factor responsible for tyrosine residues phosphorylation in STAT5 proteins. Additionally, this observation was confirmed using RNAiinduced gene silencing techniques. The mechanism of the STAT5 phosphorylation was explained and it was suggested that the JAK2 kinase might become an effective therapeutic target in AML patients without mutations in FLT3 and KIT genes. In another study, Zhang et al. [18] attempted to explain the role of FLT3 gene mutations by assessing the phosphotyrosine content in AML cells producing the wild type FLT3, the protein with internal tandem duplication (FLT-ITD) and the protein with a point mutation (FLT3 D835Y). This analysis showed that the phosphorylation of tyrosine phosphatase SHP1 abolishes the transformation potential although not totally inhibits FLT3-D835Y kinase activity. Accordingly, Zhang and coworkers concluded that both oncogenic mutations of Flt3 (FLT-ITD and FLT3-D835Y) result in the different activation of aberrant signaling pathways in AML [18].

STP proteins are another objects of intensive studies aimed at elucidating pathophysiology of acute leukemia $[19,20]$. This issue is particularly interesting and complex as in case of these proteins the changes observed at the protein level are often not reflected at the RNA level. The analysis of leukemic cells isolated from patients belonging to various subgroups [21] showed that the majority of them had an identical and undisturbed pattern of the expression of STP protein genes. For example, in all subgroups, the levels of pAKT and pS6RP kinases well correlated with the levels their substrates - GSK3 or P70S6K. Generally, STP activation is connected with poorer prognosis. It is known that AKT is an inactivator of the proapoptotic BAD protein and an activator of mTOR kinase (mammalian target of rapamycin kinase). Kornblau et al. showed that at high AKT.p473 and AKT.p308 concentrations, the levels of phosphorylated proteins - BAD.p136 and MTOR.p - are low and, conversely, the latter are high when the AKT.p473 and AKT.p308 concentrations are low. This finding revealed some atypical relationships between the levels of gene expression and the activities of resultant proteins, which may 
imply the deregulation of signal transduction systems in leukemic cells. An increased activity of AKT in leukemia usually results from the loss of PTEN phosphatase activity. However, it was observed that AKT.p308 and AKT.p473 levels in one of the subgroups are high, similarly as the PTEN level. Such a result may suggest that PTEN-dependent regulation of AKT kinase dephosphorylation is ineffective. This phenomenon seems to be an example of feedback failure when PTEN synthesis is increased [22, 23].

Disturbance of the activity of acetyltransferases and histone deacetylases (HDAC) is another pathogenic mechanism in AML. It leads to the dysfunction of systems controlling the transcription of genes involved in cell cycle regulation, differentiation and apoptosis. In acute promyelocytic leukemia $(\mathrm{APL})$, a significant element of pathomechanism is incorrect HDAC recruitment by the PML-RAR fusion protein. Acute promyelocytic leukemia therapy resulting in cell differentiation disrupts HDAC-induced suppression of retinoic acid targeted genes. These observations led to a dramatic increase of studies on the potential factors modulating the activity of proteins involved in chromatin modification, including HDAC inhibitors. They can inhibit cell development, differentiation and apoptosis both in vitro and in vivo by affecting the mechanisms dependent on and independent of transcription. Preliminary experiments showed that HDAC inhibitors may affect the level of expression of $7-10 \%$ of genes. Recently, however, it has been demonstrated that the primary HDAC activity may be also targeted directly at non-histone substrates. So far, over 1700 proteins of this type have been identified. They include transcription factors, such as GATA-1, p53 and STAT-3 as well as structural and chaperone proteins such as HSP90 [7].

In general, the results presented above indicate that mass spectrometry-based approaches to cell proteome analysis may prove to be a valuable method for tracking various biological processes, including leukemogenesis.

\section{Proteomics in acute myeloid leukemia diagnostics}

Rapid progress in research on molecular mechanisms of leukemic transformation enforces continuous modification of AML diagnostic and classification systems. Accordingly, many projects focus on protein biomarkers that can be used to differentiate $A M L$ from other types of leukemia and also to differentiate AML subtypes. The research in this area was started over 20 years ago when Hanash et al. [24] found in acute lymphoblastic leukemia (ALL) cells a group of proteins specific for particular subtypes of this disease as well as proteins differentiating ALL from AML This discovery can be considered a breakthrough, despite the fact that it was made at a time when rapid protein identification was not possible yet. Only a few years later subsequent studies reported the identification of the first potential protein biomarkers [25]. Researchers' attention was drawn to the oncoprotein-18 (Op-18) and non-metastaticprotein $23-\mathrm{H} 1$ which accumulation increased in mitogen-treated lymphocytes and in cells collected from AML patients [26]. It was also found that an increase of Op-18 concentration correlated with the level of this protein's phosphorylation. Accordingly, it was suggested that Op-18 might be involved in signal transduction during AML development [27]. Recently, Shi et al. have found that the platelet factor 4 , the connective tissue-activating protein3 (CTAP-III) and two fragments of the C3a complement system are potential protein biomarkers of ALL in children. These biomarkers can be used to differentiate ALL patients from both AML patients and from healthy persons [28].

In addition, proteins which are potential biomarkers differentiating AML from the myelodysplastic syndrome (MDS) were identified. For example, Aivado et al. postulated that serum proteins CXCL4 (their other name is platelet factor-4 - PF4) and chemokine (C-X-C motif) ligand 4 and 7 (CXCL4 and 7) are specific biomarkers differentiating MDS and AML patients [29]. Braoudaki et al., on the other hand, found that MOES, EZRI and AIFM1 proteins should be considered as AML biomarkers [30].

Research on protein biomarkers usually involves cell lines or leukemic cells collected from patients. Plasma is also a very useful material for such analyses, as it is an excellent source of protein biomarkers. 2DE-MS analyses have shown that eight proteins (including ATPases, subunits of $26 \mathrm{~S}$ proteasome and a fragment of haptoglobin-1) accumulate to a higher concentration in plasma of AML patients than in plasma of healthy persons [31]. Using the same methods, proteomes of stem cells (with the $\mathrm{AC} 133^{+}$antigen) isolated from AML patients and healthy persons were also compared. As a result, the following proteins characteristic of AML cells were identified: nuclear mitotic apparatus protein (NuMA), heat shock proteins and redox regulators [32].

Due to the heterogeneity of $\mathrm{AML}$, finding new biomarkers enabling its better classification is another important goal of proteomic analyses. Few articles devoted to this issue have been published so far. In one of them, Cui et al. [33] presented a comparative 2DE-MS analysis of bone marrow mononuclear cells collected from 61 patients diagnosed with various AML subtypes (FAB). As a result, 23 proteins specific for the individual AML subtypes were identified, including proteinase 3, azurocidin and cation antimicrobial peptides which accumulated to high levels in AML-M2 and AML-M3 patients. In another study, $13 \mathrm{AML}$ patients classified according to the WHO system were analyzed [34]. Interestingly, authors of this studies identified a completely different set of proteins specific for AML subtypes, including: $\alpha$-enolase, Rho-GDI $\beta$-protein, annexin I and X, catalase, peroxiredoxin 2 and tropomyosin 3. A very small studied group was a significant weakness of this analysis - each AML subtype was represented by only one or two patients. Yet another type of AML patients classification was examined by Balkhi et al. [35]. These authors attempted to find protein biomarkers characteristic for typical chromosomal aberrations observed in AML. As a result, they determined that transcription factor MafK is characteristic for patients with inv(16), the $\mathrm{H} 17$ variant of myeloperoxidase for patients with $\mathrm{t}(15 ; 17)$ and sorcin for patients with t(8;21) [35]. In 2009, Kornblau et al. applied protein microarrays to determine differences between proteomes of AML cells. They identified a set of 24 proteins which can be used to distinguish between purely myeloid subtypes of leukemia (MO, M1, M2) and those with the monocytic components (M4, M5). Additionally, the analyses conducted by Kornblau and coworkers showed that all these AML subtypes (M0-5) differ from the remaining two FAB subtypes, i.e. AML 
M6 (erythroleukemia) and AML M7 (megakaryotic leukemia). For example, the concentrations of the phosphorylated STAT1 and STAT5 proteins as well as a few proteins connected with apoptosis were higher in AML, MO, M1 and M2 than in M4 and M5. Conversely, other phosphorylated signal transduction proteins (AKT, TP38, PKC and SRC.p527) accumulated to the higher levels in subtypes with the monocytic component [21].

Proper classification of AML patients still poses a lot of difficulty. Persons with the same result of cytogenetic tests, with identical genetic disorders and with similar risk factors, can respond to therapy in quite different ways. Hence, the identification of factors enabling the individualization of AML treatment has become another important challenge for proteomics. Accordingly, Irish et al. [36] employed multiparameter flow cytometry to determine in a single $A M L$ cell the changes in protein phosphorylation that are induced by chemotherapy. They observed that the phosphorylation of some important elements of signal transduction pathways (STAT proteins and mitogen-activated protein kinases (MAPK)) is associated with patient response to treatment. As a result Irish and coworkers distinguished four phosphoprotein profiles that correlated with the prognosis and response to the treatment, but not with the cytogenetic disorders. Kornblau et al. [21] proposed yet another approach to the classification of acute myeloid leukemia. The proteins that could be analyzed by microarray analysis were divided into 10 separate, interconnected "constellations". Each of them comprises proteins with a similar function. After the experiment the accumulation of all proteins within each constellation was summarized. Consequently, every sample was characterized by 10 numbers corresponding to 10 constellation. As a result, the authors distinguished 7 subgroups within AML patients. This finding proved the exceptional potential of proteomic research as a source of information, which cannot be obtained with other classic or modern genomic methods. It should be remembered, however, that these results often have a very preliminary character, so they require further confirmation.

\section{Protein biomarkers for acute myeloid leukemia remission, resistance and relapse}

The identification of protein biomarkers, which can find applications in $\mathrm{AML}$ prognostics, requires comparative analyses of bone marrow or blood cell proteomes collected from AML patients before treatment, after treatment and when the disease relapses. Unfortunately, such research is very rare due to the problems with obtaining appropriate material for proteomic analyses. It is known that chemotherapy results in the eradication of cancer cells and considerable myelosuppression. Despite treatment, it is not possible to eliminate all leukemic cells in some patients. In addition, noneliminated leukemic cells can proliferate intensively and initiate relapse. The few studies comparing three major AML stages suggest that the immunophenotype of leukemic cells at the beginning of the disease and during its relapse can be different. Such changes were observed both in adult persons [37] and in children suffering from AML [38]. A comparative analysis of proteomes isolated before and after treatment showed a higher accumulation of BTG1 suppressor protein (BTG1 - B-cell translocation gene 1) in cells collected from $A M L-M 2$ and $-M 3$ patients with complete remission [39]. Additionally, Western blot analyses of various heat shock proteins (HSP) confirmed that in patients with medium and high risk there is a correlation between an increase of HSP concentration and the unfavorable course of the disease. This observation showed that HSPs play a significant role in both the apoptosis and AML resistance to chemotherapy [40]. The so-called protein profiles characterizing the proteome composition in AML can also be useful for predicting disease outcome. The developed patterns imply, for example, that the lack of response to treatment in AML-MO, - M1 and-M2 depends, to a large extent, on the resistance to apoptosis. The blast growth and resistance to therapy in AML-M4 and -M5, on the other hand may be connected with the transduction of signals inducing proliferation and antiapoptotic reactions [21]. Similarly, some differences in protein profiles seem to be characteristic for typical cytogenetic disorders observed in AML. Considering the current knowledge, it can be concluded that patients with the disorders of chromosomes 5 and 7 respond poorly to treatment and their survival is very short. Advanced proteomic analyses of leukemic cells showed significant similarities in protein profiles in patients with monosomy of chromosomes 5,7 ( $-5,-7$, respectively) or deletion in the long arm of chromosome 5 (5q) as well as in patient with complex chromosomal aberrations, e.g. simultaneous monosomy of chromosomes 5 and 7, or simuItaneous monosomy of chromosomes 5 and 7 accompanied by trisomy of chromosome $8(+8)$. Analyses of the gene expression profiles [6] led to identical observations. These results show that various cytogenetic changes may have a similar influence on proteome composition and leukemic blast activation. In patients with cytogenetic disorders -5 and -7 , an increase of survivin and GSK3 concentrations was also observed. Survivin is a poor prognostic marker at the advance stages of AML [41]. Increased concentrations of neuropilin-1, semaphorin receptor and mutated forms of $\mathrm{p} 53$ protein are also considered as an unfavorable prognostic factors [42, 43]. An increased accumulation of MCL1 protein, on the other hand, seems to be connected with resistance to AML treatment [44].

\section{Proteomics in the assessment of acute myeloid leukemia patients' response to treatment}

Proteomic analyses play a very important role in the identification of both potential therapeutic targets and treatmentinduced changes in human cells. Thus, proteomic methods also found application in the studies on mechanisms of the action of drugs used in AML chemotherapy. For example, daunorubicin-induced changes in leukemic cells were analyzed. It was found that the administration of this antracyclin cytostatic drug caused the protective proteins p23 and HSP90 complex to be degraded by caspases. Similar effects were observed if idarubicin, cytosine arabinoside and etoposide were used. As a result it was hypothesized that inhibition of the p23 protein may play a significant role in the search of new targets in AML therapy [45]. The increased HSP9O concentration in AML cells had been shown previously. Accordingly, HSP9O became the object of intensive research as a po- 
tential therapeutic target [46]. One of the functions of the HSP9O chaperone complex is controlling and supporting the proper folding of, among other things, tyrosine kinase receptors c-Kit [47] and FLT3 (fms-like tyrosine kinase 3) [48]. Following these clues, researchers have developed HSP9O inhibitors: geldanamycin and 17-allyl-amino-geldanamycin. These compounds are currently tested $[49,50]$. The results obtained so far are very promising as cancer cells exhibit a much higher affinity to these inhibitors than healthy cells [51]. In addition, it turned out that the application of a geldanamycin derivative increases the sensitivity of cancer cells to cytarabine, i.e. one of the cytostatics currently used [52].

A similar pattern of action was used for searching the receptors of all-trans-retinoic acid (ATRA) in acute promyelocytic leukemia (APL). The application of ATRA induced leukemic cell differentiation to mature forms of granulocytes. As a result, complete remission was obtained in about 70\% of APL patients [53]. ATRA binds with fusion protein PML-RAR $\alpha$ (promyelocytic leukemia-retinoic acid receptor $\alpha$ ) and induces its decomposition. Consequently, the genes needed for cell differentiation and apoptosis become reactivated [54]. As the molecular mechanism underlying ATRA activity was not wellunderstood, numerous reports describing this issue appeared over a short period of time. Some authors attempted to solve the problem using proteomic techniques. In one such study, proteins whose concentrations change under ATRA treatment were identified. This group included three isoforms of the 14-3-3 protein which activate protein kinase C (PKC). It was found that their decreased accumulation induces the cascade of signals that activate of caspase protein family. As a result, apoptosis is induced and the development of APL is inhibited. The mechanism postulated based on the proteomic studies (2DE and MS) was then confirmed with classical techniques, mostly Western blot [55]. These analyses showed that ATRA affects the accumulation of several other proteins. The increased concentration of coronine, an actinbinding protein, pyruvate kinase and glutathione transferase were observed. Unfortunately, the observations made using 2DE-MS are not fully consistent with the results of DNA microarray analyses [56].

Arsenic trioxide (ATO) is another factor that induce partial differentiation and apoptosis of APL cells. Xiong and Wang used proteomic methods to determine the mechanisms of cytotoxic and therapeutic activities of ATO [57]. Attempts to use both drugs simultaneously, i.e. ATRA and ATO, brought a quite good results and patient mortality rate was reduced [58]. The combination of proteomic and genomic analyses and the application of computational biology methods made it possible to identify other genes and proteins whose activity is modulated by one or both drugs. At the same time, signal pathways affected by ATO and ATRA were identified [59]. Moreover, it was found that in ATRA-resistant patients better therapeutic effect can be obtained by application of ATRA together with inhibitors of histone deacetylase. Histone acetylation is one of the basic mechanisms enabling the modulation of gene expression and, thus, differentiation of cells and their maturation. Two key enzymes involved in histone modification are acetyltrasferase and histone deacetylase. Histone deacetylase inhibitors stimulate differentiation and/or selective apopto- sis of cancer cells, so they are a very promising potential therapeutics (e.g. trichostatin A, sodium butyrate and valproic acid). Bartels et al. examined their influence on the development of myeloid line, its potential to form colony, proliferation and differentiation of neutrophils. They found that trichostatin A gently reduced the proliferation of progenitor cells, while sodium butyrate and valproic acid affected their proliferation and apoptosis. Moreover, valproic acid stimulated the proliferation of $\mathrm{CD}^{34+}$ cells. Additionally, sodium butyrate inhibited neutrophil differentiation. The administration of $100 \mu \mathrm{M}$ of valproic acid increased the number of mature neutrophils with a differentiation block. Sodium butyrate and valproic acid increased the acetylation of histones 3 and 4 . Moreover, all three tested substances (trichostatin A, sodium butyrate and valproic acid) affected the acetylation of non-histone proteins [7].

For many years, researchers' attention was focused on GTP-binding proteins and RAS proteins. Mutations in their genes are found in $15-25 \%$ of patients suffering from AML. It was found that some of these mutations could activate the RAF/MEK/ERK kinase pathway. One of numerous postulated treatment strategies involves the application of the mitogen extracellular kinase (MEK) inhibitor which should block extracellular signal-regulated kinase (ERK). Research on two MEK inhibitors showed that they protected against the abnormal activation of the RAF/MEK/ERK signal pathway. Both inhibitors repress the development and formation of colonies of numerous leukemia lines, at the same time exhibit low toxicity to normal $C D^{34+}$ cells $[60,61]$. Hahn et al. [13] found that spleen tyrosine kinase (SYK) could also be a good target in AML therapy. This protein belongs to the so-called nonreceptor kinases and it takes part in the differentiation of $B$ lymphocytes, signal transduction and it seems to be of particular importance in lymphomas and myelodysplastic syndromes. It was found that genetic and pharmacological SYK inactivation with gefitinib promote differentiation of AML cells and reduce the in vivo development of leukemia [13].

One of the latest strategies in cancer treatment involves inhibition of activity of the ubiquitin-proteasome system. Proteasome 26S is an enzymatic complex present in the nucleus and cytoplasm of each cell. It plays a vital role in protein degradation and in this way participates in the cell cycle and transcription regulation. Degradation of proteasome proteins activates other factors significant for cell development. Inhibitors of individual proteasome components constitute a large number of drugs currently used or tested. This group includes peptide aldehyde (MG132), dipeptidyl boronic acid (PS-341), tripeptide vinyl sulfate (NLVS), epoxomycin and lactacystin. The influence of MG132 and lactacystin on the composition of leukemic cell proteome was examined using 2DE and MS techniques. It was found that the proteasome inhibitors affected the accumulation of 39 different proteins, including 11 proteins connected with apoptosis [62]. Agarwal et al. observed that the administration of methotrexate increased the accumulation of ubiquitinated proteins in a $\mathrm{HL}-60$ cell line. The result indicated significant disturbance of proteasome function [63].

Yet another strategy for the cancer treatment is based on specific antigens of transformed cells. Therapies involving monoclonal antibodies have already been successfully used 
in solid tumor treatment. The first reports describing the application of this strategy in AML therapy have already appeared [64]. Specific antibodies which induced a humoral response in leukemic cells were detected by proteomic (2DE, MS) and immunodetection methods. Antibodies against the inhibitor of Rho GDP dissociation, actin-binding protein, CAPZA1 and g-actin were identified. The antibodies against the inhibitor of Rho-GDP dissociation were found in $71 \%$ of AML patients, while in case of solid tumors and in healthy controls, these antibodies were detected only in 5 and $4.5 \%$ of persons, respectively. The individual antigens may, therefore, constitute both diagnostic markers and therapeutic targets also in AML treatment.

Very interesting results of the proteomic analyses have been recently published by Tsao et al. [14]. They showed an elevated accumulation of peroxisome proliferator-activated receptor $\gamma$ (PPAR $\gamma)$ in 260 newly diagnosed AML patients. PPAR $\gamma$ belongs to the family of transcription factors playing an important regulatory role in cell development, differentiation and apoptosis. Increased expression of the PPAR $y$ transcription factor gene induced its antagonists, thus increasing the sensitivity of myeloid leukemia cells to apoptosis by caspase-8 decomposition [14].

In conclusion: the dynamic development of modern proteomic methods observed in recent years has opened new perspectives in the research on mechanisms underlying cancerogenesis, including leukemic transformation. Special attention is paid to the identification of new biomarkers that ensure more effective diagnostics, treatment monitoring and therapeutic outcome prediction. Accordingly, more and more frequently proteomes (complex systems formed by proteins that interact with one another and with other cell components) are the object of medical studies.

One of the major challenges faced by proteomics is its integration with other fields of biological sciences, especially with genomics, metabolomics, lipidomics, glycomics and transcriptomics. Proteomics by itself is not sufficient to explore the mechanisms shaping extremely complicated biological phenomena such as cancer. The replacement of reductionist methods and research approaches with holistic methods and approaches appears to be the key to success. In addition, some attempts are being made to introduce proteomics into clinical diagnostics in the broad sense $[65,66]$. Researchers dealing with this problem pay particular attention to the necessity of selection, verification and determination of the so-called standard operational procedures (SOP). They obtain repeatable and clinically useful results by standardization of the conditions under which samples are obtained (material collection, transport and preparation directly before the analysis). The optimization of clinical tests is another issue. It requires the introduction of high-performance analytical platforms to obtain repeatability of results. These tests must not only be efficient, but also easy to use; they have to require minimum supervision and have to be easy to introduce in a clinical laboratory. It is also necessary to develop computational methods which would transform numerous proteomic results into clinically useful data that could be integrated with hospital computer systems quickly and easily. Moreover, a system of biobanks and biorepositories should be created and all formal and legal aspects of the research should be regulated (patients' consent, commercial use of samples, etc.), preferably at an international level.

Chronic myeloid leukemia (CML), which together with $\mathrm{AML}$ is included among the myeloid diseases, is the best example of a versatile use of proteomics in clinical trials. It is possible that CML will become the first leukemia for which constant diagnostics and monitoring of therapy will be introduced. As a result of the discovery of the association between $\mathrm{t}(9 ; 22)$ and fusion BCR-ABL protein production, a drug specifically inhibiting the activity of $A B L$ tyrosine kinase was created. At present, tests for BCR-ABL protein detection are being prepared. They will be used to diagnose leukemia (BCR/ABL positive) based on single cell proteome analysis or single cell phenotype assessment in flow cytometry. Therefore, analyses currently used in CML diagnostics will in future function solely as confirming tests [67].

Chronic myeloid leukemia (CML), which together with $\mathrm{AML}$ belongs to myeloid diseases, is the best example of a versatile use of proteomics in clinical trials. It is possible that CML will become the first leukemia, for which constant diagnostics and monitoring of therapy will be introduced. As a result of the discovery of the association between the $t(9 ; 22)$ and fusion BCR-ABL protein production, a drug specifically inhibiting the activity of $A B L$ tyrosine kinase was created. At present, the tests for BCR-ABL protein detection are being prepared. They will be used to diagnose leukemia (BCR/ABL positive) based on a single cell proteome analysis or a single cell phenotype assessment in flow cytometry. Therefore, analyses currently used in CML diagnostics will in future function solely as confirming tests [67].

Preparation of this article was supported by the Polish Ministry of Science and Higher Education grant No. PBZ_Mnil2/1/2005.

\section{References}

1. Bennet JM, Catovsky D, Daniel MT. Proposals for the classification of the acute leukaemias. French-American-British (FAB) co-operative group. Br J Heamatol 1976; 33: 451-8.

2. Bennet JM. World Health Organization classification of the acute leukemias and myelodysplastic syndrome. Int J Hematol 2000; 72: 131-3.

3. Hanahan D, Weinberg RA. The hallmarks of cancer. Cell 2000; 100: 57-70.

4. Fresno Vara JA, Casado E, de Castro J, Cejas P, Belda-Iniesta C, Gonzalez-Baron M. PI3K/Akt signalling pathway and cancer. Cancer Treat Rev 2004; 30: 193-204.

5. Bullinger L, Dohner K, Bair E, Fröhling S, Schlenk RF, Tibshirani R, Döhner $\mathrm{H}$, Pollack JR. Use of gene expression profiling to identify prognostic subclasses in adult acute myeloid leukemia. N Engl J Med 2004; 350: 1605-16.

6. Valk PJ, Verhaak RG, Beijen MA, et al. Prognostically useful gene-expression profiles in acute myeloid leukemia. N Engl J Med 2004; 350: 1617-28.

7. Bartels M, Geest CR, Bierings M, Buitenhuis M, Coffer PJ. Histone deacetylase inhibition modulates cell fate decisions during myeloid differentiation. Haematologica 2009; 95: 1052-60.

8. Pabst T, Mueller BU, Zhang P, et al. Dominant-negative mutations of CEBPA, encoding CCAAT/enhancer binding protein-alpha (C/EBPalpha), in acute myeloid leukemia. Nat Genet 2001; 27: 263-70.

9. Gombart AF, Hofmann WK, Kawano S, et al. Mutations in the gene encoding the transcription factor CCAAT/enhancer binding protein alpha in myelodysplastic syndromes and acute myeloid leukemias. Blood 2002; 99: 1332-40. 
10. Geletu M, Balkhi MY, Peer Zada AA, Christopeit M, Pulikkan JA, Trivedi AK, Tenen DG, Behre G. Target proteins of C/EBP\{alpha\}p30 in AML: C/EBP\{alpha\}p30 enhances sumoylation of C/EBP\{alpha\}p42 via upregulation of Ubc9. Blood 2007; 110: 3301-9.

11. Zada AA, Pulikkan JA, Bararia D, et al. Proteomic discovery of Max as a novel interacting partner of C/EBP $\alpha$ : a Myc/Max/Mad link. Leukemia 2006; 20: 2137-46.

12. Pulikkan JA, Dengler V, Peer Zada AA, et. al. Elevated PIN1 expression by C/EBPalpha-p30 blocks C/EBPalpha-induced granulocytic differentiation through c-Jun in AML. Leukemia 2010; 24: 914-23.

13. Hahn CK, Berchuck JE, Ross KN, et al. Proteomic and genetic approaches identify Syk as an AML target. Cancer Cell 2009; 16: 281-94.

14. Tsao T, Kornblau S, Safe S, et al. Role of peroxisome proliferatoractivated receptor-gamma and its coactivator DRIP205 in cellular re sponses to CDDO (RTA-401) in acute myelogenous leukemia. Can cer Res 2010; 70: 4949-60.

15. Hayakawa F, Towatari M, lida H, Wakao H, Kiyoi H, Naoe T, Saito H. Differential constitutive activation between STAT-related proteins and MAP kinase in primary acute myelogenous leukaemia. Br J Haematol 1998; 101: 521-8.

16. Birkenkamp KU, Geugien M, Lemmink HH, Kruijer W, Vellenga E. Regulation of constitutive STAT5 phosphorylation in acute myeloid leukemia blasts. Leukemia 2001; 15: 1923-31.

17. Walters DK, Goss VL, Stoffregen EP, et al. Phosphoproteomic analy sis of AML cell lines identifies leukemic oncogenes. Leukemia Res 2006; 30: 1097-104

18. Zhang Y, Askenazi M, Jiang J, Luckey CJ, Griffin JD, Marto JA. A robust error model for ITRAQ quantification reveals divergent signaling between oncogenic FLT3 mutants in acute myeloid leukemia. Mol Cell Proteomics 2010; 9: 780-90.

19. Kornblau SM, Vu HT, Ruvolo P et al. BAX and PKCalpha modulate the prognostic impact of $\mathrm{BCL} 2$ expression in acute myelogenous leukemia. Clin Cancer Res 2000; 6: 1401-9.

20. Kornblau SM, Womble M, Oiu YH, Jackson CE, Chen W, Konopleva M, Estey EH, Andreeff M. Simultaneous activation of multiple signal transduction pathways confers poor prognosis in acute myelogenous leukemia. Blood 2006; 108: 2358-65.

21. Kornblau SM, Tibes R, Qiu YH, Chen W, Kantarjian HM, Andreeff M, Coombes KR, Mills GB. Functional proteomic profiling of AML predicts response and survival. Blood 2009; 113: 154-64.

22. Cantley LC, Neel BG. New insights into tumor suppression: PTEN suppresses tumor formation by restraining the phosphoinositide 3-kinase/AKT pathway. Proc Natl Acad Sci U S A 1999; 96: 4240-5.

23. Wu X, Senechal K, Neshat MS, Whang YE, Sawyers CL. The PTEN/MMAC1 tumor suppressor phosphatase functions as a neg ative regulator of the phosphoinositide 3-kinase/Akt pathway. Proc Natl Acad Sci U S A 1998; 95: 15587-91.

24. Hanash SM, Neel JV, Baier LJ, Rosenblum BB, Niezgoda W, Markel D. Genetic analysis of thirty-three platelet polypeptides detected in twodimensional polyacrylamide gels. Am J Hum Genet 1986; 38: 352-60.

25. Strahler JR, Kuick R, Eckerskorn C, et al. Identification of two relat ed markers for common acute lymphoblastic leukemia as heat shock proteins. J Clin Invest 1990; 85: 200-7.

26. Keim D, Hailat N, Melhem R, Zhu XX, Lascu I, Veron M, Strahler Hanash SM. Proliferation-related expression of p19/nm23 nucleoside diphosphate kinase. J Clin Invest 1992; 89: 919-24.

27. Zada AA, Geletu MH, Pulikkan JA, et al. Proteomic analysis of acute promyelocytic leukemia: PML-RARalpha leads to decreased phosphorylation of OP18 at serine 63. Proteomics 2006; 6: 5705-19.

28. Shi L, Zhang J, Wu P, et al. Discovery and identification of potential biomarkers of pediatric. Acute Lymphoblastic Leukemia. Proteome Sci 2009; $7: 7$.

29. Aivado M, Spentzos D, Germing U, et al. Serum proteome profiling detects myelodysplastic syndromes and identifies CXC chemokine ligands 4 and 7 as markers for advanced disease. Proc Natl Acad Sci U S A 2007; 104: 1307-12

30. Braoudaki M, Tzortzatou-Stathopoulou F, Anagnostopoulos AK, Papathanassiou C, Vougas K, Karamolegou K, Tsangaris GT. Proteomic analysis of childhood de novo acute myeloid leukemia and myelodysplastic syndrome/AML: correlation to molecular and cytogenetic analyses. Amino Acids 2011; 40: 943-51.
31. Kwak JY, Ma TZ, Yoo MJ, Choi BH, Kim HG, Kim SR, Yim CY, Kwak YG. The comparative analysis of serum proteomes for the discovery of biomarkers for acute myeloid leukaemia. Exp Hematol 2004; 32: 836-42.

32. Ota J, Yamashita Y, Okawa K, et al. Proteomic analysis of hematopoietic stem cell-like fractions in leukemic disorders. Oncogene 2003; 22: 5720-8.

33. Cui J-W, Wang J, He K, et al. Two-dimensional electrophoresis protein profiling as an analytical tool for human acute leukemia classification. Electrophoresis 2005; 26: 268-79.

34. López-Pedrera C, Villalba JM, Siendones E, et al. Proteomic analysis of acute myeloid leukemia: identification of potential early biomarkers and therapeutic targets. Proteomics 2006; 6: S293-9.

35. Balkhi MY, Trivedi AK, Geletu M, Christopeit M, Bohlander SK, Behre HM, Behre G. Proteomics of acute myeloid leukaemia: Cytogenetic risk groups differ specifically in their proteome, interactome and post-translational protein modifications. Oncogene 2006; 25 : 7041-58

36. Irish JM, Hovland R, Krutzik PO, Perez OD, Bruserud $\emptyset$, Gjertsen BT, Nolan GP. Single cell profiling of potentiated phospho-protein networks in cancer cells. Cell 2004; 118: 217-28.

37. Baer MR, Stewart CC, Dodge RK, et al. High frequency of immunophenotype changes in acute myeloid leukemia at relapse: implications for residual disease detection (Cancer and Leukemia Group B Study 8361). Blood 2001; 97: 3574-80.

38. Langebrake C, Brinkmann I, Teigler-Schlegel A, Creutzig U, Griesinger F, Puhlmann U, Reinhardt D. Immunophenotypic differences be tween diagnosis and relapse in childhood AML: Implications for MRD monitoring. Cytometry B Clin Cytom 2005; 63: 1-9.

39. Cho JW, Kim JJ, Park SG, Lee DH, Lee SC, Kim HJ, Park BC, Cho S. Identification of B-cell translocation gene 1 as a biomarker for monitoring the remission of acute myeloid leukemia. Proteomics 2004; 4: 3456-63.

40. Thomas X, Campos L, Mounier C, Cornillon J, Flandrin P, Le QH, Piselli S, Guyotat D. Expression of heat-shock proteins is associated with major adverse prognostic factors in acute myeloid leukemia. Leuk Res 2005; 29: 1049-58.

41. Adida C, Recher C, Raffoux E, et al. Expression and prognostic significance of survivin in de novo acute myeloid leukaemia. Br J Haematol 2000; 111: 196-203.

42. Kreuter M, Woelke K, Bieker R, Schliemann C, Steins M, Buechner T, Berdel WE, Mesters RM. Correlation of neuropilin-1 overexpression to survival in acute myeloid leukemia. Leukemia 2006; 20: 1950-54.

43. Wattel E, Preudhomme C, Hecquet B, Vanrumbeke M, Quesnel B, Dervite I, Morel P, Fenaux P. p53 mutations are associated with resistance to chemotherapy and short survival in hematologic malignancies. Blood 1994; 84: 3148-57.

44. Kaufmann SH, Karp JE, Svingen PA, Krajewski S, Burke PJ, Gore SD, Reed JC. Elevated expression of the apoptotic regulator $\mathrm{Mcl}-1$ at the time of leukemic relapse. Blood 1998; 91: 991-1000.

45. Gausdal G, Gjertsen BT, Fladmark KE, Demol H, Vandekerckhove J, Doskeland SO. Caspase-dependent, geldanamycin-enhanced cleavage of cochaperone p23 in leukemic apoptosis. Leukemia 2004; 18: 1989-96.

46. Chant ID, Rose PE, Morris AG. Analysis of heat-shock protein expression in myeloid leukaemia cells by flow cytometry. Br J Haematol 1995; 90: 163-8.

47. Fumo G, Akin C, Metcalfe DD, Neckers L. 17-AAG is effective in downregulating mutated, constitutively activated KIT protein in human mast cells. Blood 2004; 103: 1078-84.

48. Yao O, Nishiuchi R, Li O, Kumar AR, Hudson WA, Kersey JH. FLT3 expressing leukemias are selectively sensitive to inhibitors of the molecular chaperone heat shock protein 90 through destabilization of sig nal transduction-associated kinases. Clin Cancer Res 2003; 9: 4483-93.

49. Nimmanapalli R, O’Bryan E, Bhalla K. Geldanamycin and its analogue 17-allylamino-17-demethoxygeldanamycin lowers Bcr-Abl levels and induces apoptosis and differentiation of Bcr-Abl-positive human leukemic blasts. Cancer Res 2001; 61: 1799-804

50. Goetz MP, Toft DO, Ames MM, Erlichman C. The Hsp90 chaperone complex as a novel target for cancer therapy. Ann Oncol 2003; 14 1169-76.

51. Kamal A, Thao L, Sensintaffar J Zhang L, Boehm MF, Fritz LC, Burrows FJ. A high-affinity conformation of Hsp90 confers tumour selectivity on Hsp90 inhibitors. Nature 2003; 425: 407-10. 
52. Mesa RA, Loegering D, Powell HL et al. Heat shock protein 90 inhibition sensitizes acute myelogenous leukemia cells to cytarabine. Blood 2005; 106: 318-27.

53. Fenaux P, Chastang C, Chevret S, et al. A randomized comparison of all transretinoic acid (ATRA) followed by chemotherapy and ATRA plus chemotherapy and the role of maintenance therapy in newly diagnosed acute promyelocytic leukemia: The European APL Group. Blood 1999; 94: 1192-200.

54. Pitha-Rowe I, Petty WJ, Kitareewan S, Dmitrovsky E. Retinoid target genes in acute promyelocytic leukemia. Leukemia 2003; 17: 1723-30.

55. Harris MN, Ozpolat B, Abdi F, et al. Comparative proteomic analysis of all-trans-retinoic acid treatment reveals systematic post transcriptional control mechanisms in acute promyelocytic leukemia. Blood 2004; 104: 1314-23.

56. Wang D, Jensen R, Gendeh G, Williams K, Pallavicini MG. Proteome and transcriptome analysis of retinoic acid-induced differentiation of human acute promyelocytic leukemia cells, NB4. J. Proteome Res 2004; 3: 627-35.

57. Xiong L, Wang Y. Quantitative proteomic analysis reveals the perturbation of multiple cellular pathways in HL-60 cells induced by arsenite treatment. J Proteome Res 2010; 9: 1129-37.

58. Shen ZX, Shi ZZ, Fang J, et al. All-trans retinoic acid/As203 combination yields a high quality remission and survival in newly diagnosed acute promyelocytic leukemia. Proc Natl Acad Sci U S A 2004; 101: 5328-35.

59. Zheng P-Z, Wang K-K, Zhang Q-Y, et al. Systems analysis of transcriptome and proteome in retinoic acid/arsenic trioxide-induced cel differentiation/apoptosis of promyelocytic leukemia. Proc Natl Acad Sci U S A 2005; 102: 7653-8.

60. Morgan MA, Dolp O, Reuter CW. Cell-cycle-dependent activation of mitogen-activated protein kinase kinase (MEK-1/2) in myeloid leukemia cell lines and induction of growth inhibition and apoptosis by inhibitors of RAS signaling. Blood 2001; 97: 1823-34.

61. Fukazawa H, Uehara Y. U0126 reverses Ki-ras-mediated transformation by blocking both mitogen-activated protein kinase and p70 S6 kinase pathways. Cancer Res 2000; 60: 2104-7.

62. Jin BF, He K, Wang HX, et al. Proteomic analysis of ubiquitin-proteasome effects: insight into the function of eukaryotic initiation factor 5A. Oncogene 2003; 22: 4819-30.

63. Agarwal NK, Mueller GA, Mueller C, Streich JH, Asif AR, Dihazi H. Expression proteomics of acute promyelocytic leukaemia cells treated with methotrexate. Biochim Biophys Acta 2010; 1804: 918-28.

64. Cui JW, Li WH, Wang J, et al. Proteomics-based identification of human acute leukemia antigens that induce humoral immune response. Mol Cell Proteomics 2005; 4: 1718-24.

65. Apweiler R, Aslanidis C, Deufel T, et al. Approaching clinical proteomics: current state and future fields of application in cellular proteomics. Cytometry Part A 2009; 75A: 816-32.

66. Apweiler R, Aslanidis C, Deufel T, et al. Approaching clinical proteomics: current state and future fields of application in fluid proteomics. Clin Chem Lab Med 2009; 47: 724-44.

67. Weerkamp F, Dekking E, Ng Y, et al. Flow cytometric immunobead assay for the detection of BCR-ABL fusion proteins in leukemia patients. Leukemia 2009; 23: 1106-17.

\section{Address for correspondence}

\section{Marek Figlerowicz}

Institute of Bioorganic Chemistry

Polish Academy of Sciences

Noskowskiego 12/14,

61-704 Poznan, Poland

tel. +48 $61852-85-03$

$\mathrm{fax}+4861$ 852-05-32

e-mail: marekf@ibch.poznan.pl

Submitted: $\quad 7.03 .2011$

Accepted: $\quad$ 13.02.2012 